\title{
Early Agriculture in World Perspective
}

\author{
Peter Bellwood
}

\section{Introduction}

The worldwide archaeological record offers many instances, dated with varying degrees of reliability, of the appearance of domesticated crops and animals and the beginnings of settled agricultural life. At present, this database indicates to the satisfaction of most archaeologists that agriculture emerged directly from a hunter-gatherer background, without external diffusion, in at least six regions of the world. These are, with approximate starting dates for pre-domestication cultivation in brackets in years BP (Before Present, AD 1950 by radiocarbon convention): the Fertile Crescent of the Middle East (11,000 BP); the middle and lower courses of the Yangzi and Yellow River basins of China (9000 BP); the New Guinea highlands (between 10,000 and 6500 BP_see Chapters 11 and 12); Mesoamerica (8000 BP); northern South America and the central Andes $(8000 \mathrm{BP})$, with perhaps more than one origin region; and the Eastern Woodlands of the USA (4000 BP). These regions are shown, with their major crops and animals, in Figure 2.1 and $2.2{ }^{1}$

Other possible regions of early agriculture, of less certain date and significance, occur in the Amazon basin (3000 BP?), sub-Saharan tropical Africa including Ethiopia, the southern Saharan Sahel zone and the northern rainforest fringes of West Africa (5000 BP), and India (5000 BP) (Bellwood 2005a). Although it is quite possible that native crops and animals were domesticated by people with an existing knowledge of cultivation and animal husbandry who entered these regions, and indeed any regions across the agricultural latitudes of the earth, current data are not always strong enough for firm statements to be made. For New Guinea, available archaeological, linguistic and genetic ${ }^{2}$ sources of evidence are sufficient to indicate that both cultivation systems and domesticated crops were developed without external stimulus, as discussed further below.

It is necessary to stress here that two separate issues are involved in all debates about where, when and why agriculture developed. One issue concerns human agricultural behaviour, expressed through systems of plant cultivation and animal husbandry: conscious, repetitive, seasonal and with landscape consequences (fields, ditches, forest clearance and so forth). The other issue is domestication itself-the genetic changes selected for by human interference in the breeding cycles of animals and plants.

1 For further reading on these broad themes see Harris and Hillman 1989; Smith 1995; Harris 1996a; Bellwood and Renfrew 2002; Bellwood 2005a; Kennett and Winterhalder 2006; Zeder et al. 2006; Denham, Iriarte and Vrydaghs 2007; Demoule 2010; Gepts et al. 2012; and Bellwood 2013.

2 Genetic data are not discussed in this chapter, but those interested in such matters can consult the human biology chapters in Pawley et al. 2005. For a review of that volume see Bellwood 2005b. 


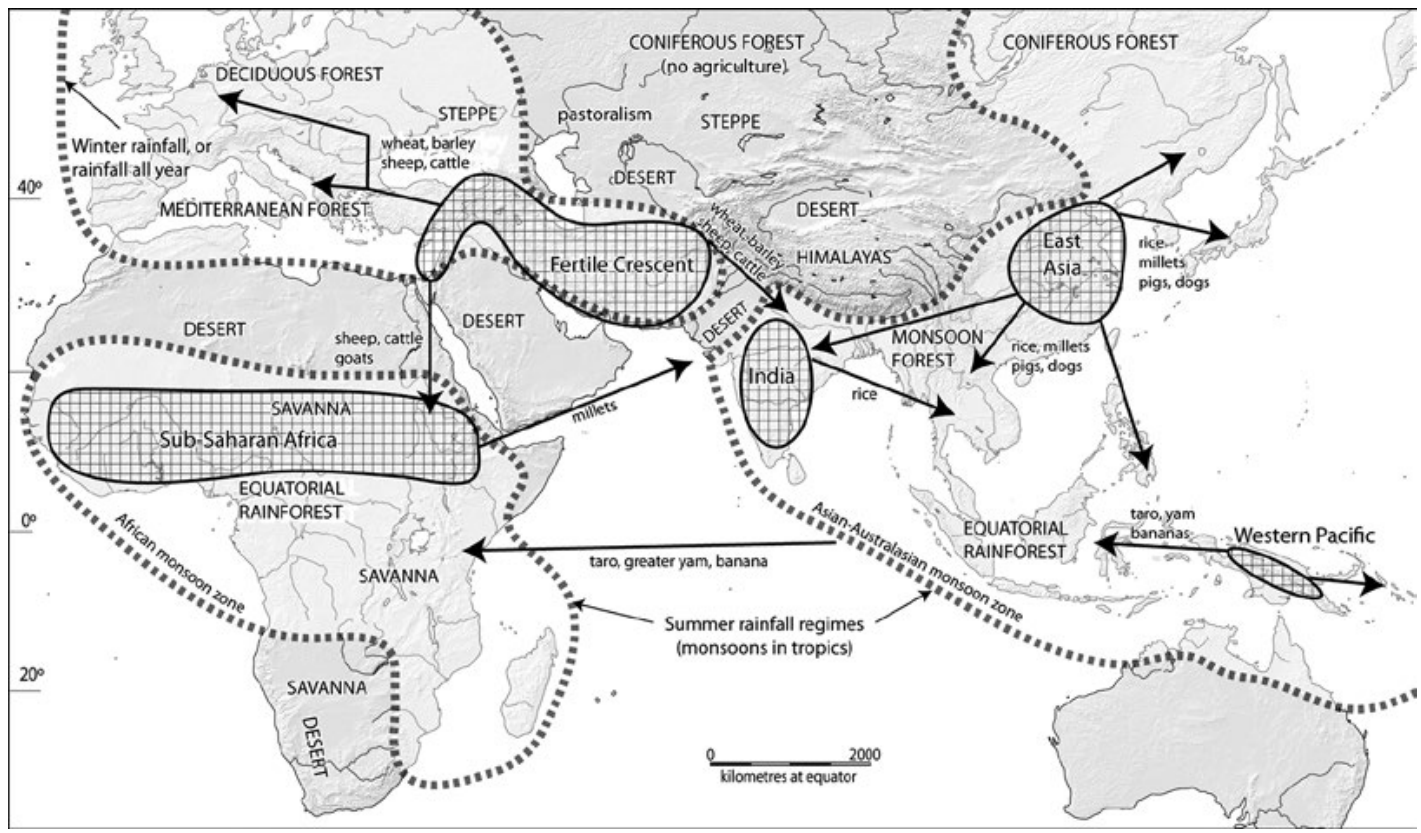

Figure 2.1 The major regions of the old World involved in the independent development of food production, with their main species of crops and animals.

Source: Drawing by Peter Bellwood (Bellwood 2013: Fig. 6.1), reproduced with permission.

Cultivation and domestication are not one and the same thing. In the Middle East, for instance, people were cultivating and transporting wild annual cereals for a millennium or more before the carbonised remains of those cereals in archaeological sites begin to reveal the basic changes in stem, seed coat and grain structure that botanists recognise as characteristic of 'domesticated' plants (Willcox 2012). This has led to recognition of a transitional situation of 'pre-domestication cultivation', often lasting for several thousand years and now documented in regions such as central China, Mesoamerica and sub-Saharan Africa (Fuller et al. 2014). Changes with domestication in the annual cereals include loss of the ability to disperse seeds through shattering when ripe, loss of seasonal seed dormancy, reduction in the thickness of protective coats around seeds, as well as increase in seed size and the development of synchronous ripening of seed heads. For animals they include reductions in body size and increases in yields of secondary products such as wool and milk.

However, the vegetatively reproduced tubers and fruits that supported New Guinea agriculture did not undergo such visible morphological changes. Instead, evidence for the presence and potential domestication of plants like bananas and taro is based more on frequency changes through time in the occurrences of pollens, microscopic silica-skeleton phytoliths and starch grains in archaeological sites and environmental cores. Kuk is an excellent example of the application of such research. Even so, the basic historical issues worthy of debate-where, when and why did people begin to cultivate?-remain the same. 


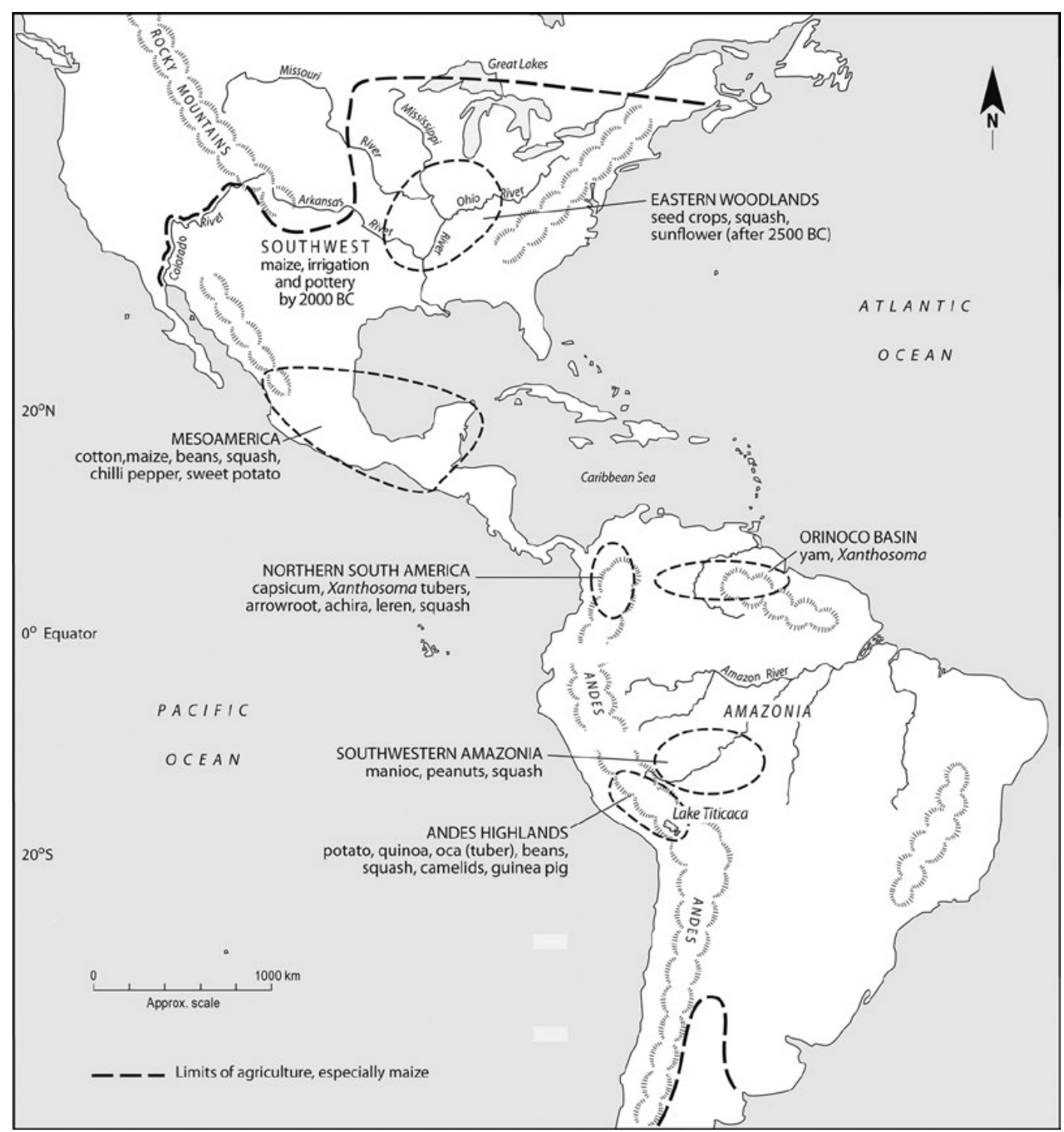

Figure 2.2 The major regions of the New World involved in the independent development of food production, with their main species of crops and animals.

Source: Drawing by Peter Bellwood (Bellwood 2013: Fig. 9.4, South American data from Piperno 2011), reproduced with permission.

Leaving New Guinea aside for the moment, of the major regions of early agriculture, we look to the Middle East for the origins of wheat, barley, sheep, goats, pigs, cattle and certain legumes (podded plants like peas and lentils); to China for the origins of rice, foxtail millet, soybean, pigs and perhaps dogs and chickens; to Africa and India for cattle, legumes, rice and several species of millets; and to various regions of the tropical Americas for maize, manioc, beans, squashes, tomatoes, potatoes and animals such as turkeys, guinea pigs, llamas and alpacas. These are only some of the major species - the minor ones would make a very lengthy list indeed. The list also indicates that some animals in Eurasia, such as pigs and cattle, and some plants, especially beans and squashes in the Americas, were domesticated more than once. However, as noted above, this need not mean that every domestication of a plant or animal species must be associated with a totally independent transition to agriculture from a foraging background. Many of these multiple domestications may have occurred as farmers moved into new regions and naturally paid attention to local wild species and their usage by local hunter-gatherers. 


\section{How did agriculture begin?}

In order to understand the specifics of agricultural origins in each of the homeland regions, including New Guinea, we must consider a number of botanical/zoological, environmental and archaeological questions:

1. What habitats contained the wild progenitors of the major domesticated plants and animals when domestication first occurred (bearing in mind that modern environments and species distributions might be very different from those of 10,000 years ago)?

2. How did climatic conditions and environments change during the time span within which agriculture emerged in the various regions? What were the results of these changes on faunas, floras and human economies?

3. What kinds of hunting and gathering cultures made the transition to agriculture in the various regions? How important were preexisting patterns of sedentism, food storage and population density?

4. How rapidly did each transition occur and over how large a region initially?

For New Guinea, answers to these four questions will not come easily, particularly for the archaeological record before the agricultural utilisation of Kuk Swamp. However, there are reasonably detailed answers for the Middle East, the best-known area of the world from this perspective and also the one that witnessed the oldest and most significant transition to agriculture, followed very closely by China. The transition to agriculture in Western Asia was based on local resources of wild annual cereals, legumes (peas and lentils) and wild ruminants such as sheep and goats. The original distributions of these species can be reconstructed in reasonable detail (Willcox 2005). The transition occurred from a baseline of relatively sedentary Natufian hunter and gatherer communities around 11,500 BP, when climates attained levels of warmth, reliable winter growing-season rainfall and long-term stability much greater than conditions typical of the immediately preceding Pleistocene glaciation; a correlation that, in my view, was not coincidental. For instance, Richerson, Boyd and Bettinger (2001) provide a compelling argument for regarding the post-glacial global warming (from c. 15,000 years ago) as a crucial factor in the origins of agriculture.

Climate alone does not explain the development of agriculture-if it did, then the whole world within agricultural latitudes would have undergone the transition in unison, and this did not happen. In the Middle East, the sheer concentration of domesticable annual plants and herd animals capable of massive yields of food and other products undoubtedly helped. Many archaeologists believe that the transition in the Middle East reflected risk management just before the phase of maximal Holocene climatic stability and/or the existence of social competition as an inducement to increased food production in growing sedentary communities. In fact, we do not really know why farming began anywhere in the world and it is unlikely that there was ever a single worldwide cause.

As far as the Middle East is concerned, agriculture developed initially, and rapidly, in an overall region of quite limited extent, but possibly with multiple minor foci of innovation, covering the lands at the eastern edge of the Mediterranean and their extension into southeastern Turkey. The transition to agriculture probably involved a shift from the harvesting and replanting of wild cereals, while still slightly unripe or 'green' (a stage at which they will not shatter and lose their seeds on harvest), to a harvesting of fully ripe grain. The resulting selection pressures on the plant genotypes would, with continual replanting of stored seed from the previous harvest, eventually 
have produced the altered features that we term 'domesticated'. As for the humans, they grew rapidly in numbers, ${ }^{3}$ as did their domesticated plants and animals, and developed a remarkably fast-spreading propensity for sedentary village life and associated cultural habits.

In understanding the origins of agriculture in all of the six or more homeland regions, we need to observe that the key to success was the practice of cultivation with replanting, usually in a specially prepared plot that then had to be guarded against pests while the crop was ripening. As noted, morphological (genetic) domestication of plants and animals was a logical result of cultivation and husbandry. In most regions for which good evidence exists, this occurred later than the initial evidence for human production of cereal crops (often by several millennia). The first cultivated crops were wild in a genetic sense, but as selection pressures increased with human manipulation, they began to change their visual appearances and growth habits. Some have even become totally dependent on human management for survival, for instance some modern cereals such as maize (sweet corn) can no longer disperse their seeds by natural means when ripe. The relationship between humans and their domesticated food species is truly one of mutual dependence.

\section{What happened in the interval between full foraging and full farming?}

The above narrative will perhaps give the impression that the transition to agriculture was a fairly straightforward progression with few complications. Being ever optimistic and concerned with big pictures rather than minutiae, I normally tend to see it thus. Some of my colleagues do not. Why?

Clearly, some hunter-gatherers as ethnographically observed can be said to impose selective pressures on the species they exploit and occasionally to indulge in activities akin to cultivation (replanting, protection and the like). This means that hunters and farmers are not like chalk and cheese, ever separate in behaviour. All agriculturalists hunt and gather if and when they canwe still go fishing in the wild today and some people in modern urban societies are involved in recreational hunting practices. Hunters and gatherers can also exploit domesticated species that they acquire from adjacent farmers. Bruce Smith (2001) has coined the term 'low-level food production' to refer to such combinations of hunting, gathering and minor exploitation of domesticated crops and animals. If we apply such observations from the recent past to interpretations of deeper prehistory, they might give the impression that the development of successful food production was a drawn-out and very gradual process, in which populations laboriously increased their dependence upon produced food, perhaps by a few per cent per millennium. ${ }^{4}$ I have doubts about such interpretations.

This is because there are important issues of historical contingency about low-level food production that must be considered. If we examine the ethnographic record of the past two centuries or so, we find that very few tribal populations existed in economies where huntinggathering and farming were evenly mixed. This is also true of New Guinea, outside the Sepik Basin. ${ }^{5}$ Furthermore, some ethnographic hunter-gatherer groups who followed such practices, for instance in the Great Basin of North America, did so because their ancestors were formerly

3 See Bocquet-Appel and Naji 2006 and Bellwood and Oxenham 2008 for discussion of increases in early farmer birth rates from skeletal and demographic perspectives.

4 One of the most significant reconstructions of this kind is for the origins of food production in Mesoamerica, derived from 1960s cave research in the Tehuacan Valley in Puebla (MacNeish 1967: Fig. 186).

5 See Murdock 1967 for documentation of ethnographic economies around the world. Roscoe 2002 discusses the Sepik populations who exploit wild stands of sago palms, as well as some domesticated fruits and tubers. 
farmers in regions that became marginal for agriculture and so had to turn to hunting and gathering to survive. ${ }^{6}$ Low-level food production only existed in agriculturally marginal circumstances where more efficient food producers could not compete, or where former food producers had switched to increased foraging in difficult environmental circumstances.

In my view, while early farmers had to undergo some kind of low-level food production transition from foraging into farming, the successful groups did not remain in this 'middle ground' situation for very long. 'Very long', of course, is a relative concept and I have already noted that domesticated crops perhaps took one or two millennia to develop from wild ones. But 2000 years is not a long time in the total span of human evolution, little more than a blink within a 50,000 year radiocarbon chronology. For New Guinea we still have very few data to throw light on this issue, especially in the early millennia of the Holocene. My working hypothesis would be that once a lifestyle based on cultivation had developed in the highlands, the momentum towards increasing dependence on cultivation would have grown rapidly. Richerson, Boyd and Bettinger (2001: 395) have used the graphic term 'competitive ratchet' to describe such situations. Once one group obtained a demographic advantage as a result of adopting systematic cultivation and planting, others would have followed rapidly, if only to maintain a status quo. New Guinea highlands ethnography reveals an intense level of competition that can hardly have developed since outsiders discovered the area in the 1930s. It is surely a fundamental human characteristic, here as amongst most other agricultural communities.

Because there is a lot of potential controversy over these issues, I think it is necessary to state clearly what my perspective on early agriculture involves, since it is likely to be very different from that presented in some other chapters of this book. I regard agriculture (with animal husbandry) as a relatively rare human development (hence the six, and perhaps a few more, regions referred to above) that, once developed, had remarkable abilities to expand. My reasons for taking this view depend not just on the archaeological record, but also on the historical implications of the subgrouping patterns within language families (a highly significant indicator of past human activity, but one ignored by many archaeologists) and the implications of human genetics research. My view is that agriculture spread mainly with its practitioners, rather than by universal in situ adoption by hunter-gatherers. I also regard language history as a more significant guide to recent human population history than the rather mute artefacts recovered by archaeologists. This is especially true for New Guinea, where regional stylistic patterns in the shapes and decorative features of artefacts do not survive in the archaeological record as richly as they do in some other archaeological homeland regions. Of course, this is not to suggest that such patterning was never present- the issue is largely one of survival of a perishable organic and aceramic material culture in a wet tropical environment.

Thus, the spread of farming in many, perhaps most, regions of the world went hand-in-hand with the spread of farmers and their languages (Diamond and Bellwood 2003; Bellwood 2005a, 2009). The significance of this observation will be reinforced below, since it might also be applicable to the New Guinea highlands situation, whether viewed from the perspective of genetics, Trans New Guinea languages or environmental significance. Admittedly for New Guinea, where cereals were not grown, the elements of causation could have been quite different from those in regions such as the Middle East and China. We simply know too little for dogmatism to prevail. But that should not stop speculation, to which I turn below.

6 For examples of this phenomenon in the Great Basin of North America see Hill 2002 and Bellwood 2005a: 37-39. Many former Fremont maize farmers in Utah eventually became hunter-gatherers as conditions for maize growing declined after AD 1300 . 


\section{How does New Guinea fit into the picture of early agriculture?}

The general region of Southeast Asia and New Guinea has given us a number of domesticated tubers, fruits and starch sources, such as coconuts, bananas, sugarcane, several species of yams and aroids (e.g. taro), breadfruit and sago. Some of the islands of Southeast Asia, from Sumatra to as far east as Sulawesi, also have native pig species and must therefore have contributed to the history of pig domestication. Yet this region was never involved with any significant indigenous domestication of cereals-rice and foxtail millet were introduced from China via Taiwan (Bellwood 2005a) — and, as far as New Guinea is concerned, no domesticated animals arrived until the pig, in association with the appearance of the Lapita culture in the Bismarck Archipelago shortly before 3000 years ago (Spriggs 1997: 88; on the dating see Summerhayes 2010: 13-24). Even so, the only evidence for a truly early and pristine origin of agriculture (devoid of any outside stimulus) in the western Pacific region comes from the New Guinea highlands. So far, there is absolutely no evidence to suggest that farming developed anywhere in Island Southeast Asia, where Neolithic populations with material assemblages deriving from the north (mainly southern China and Taiwan) first appeared around 4000 to 3500 years ago, absorbing and eventually replacing the material cultures of earlier foraging peoples. ${ }^{7}$

The discovery of early agriculture in the highlands of New Guinea came as a great surprise to many archaeologists, who had become accustomed to finding such phenomena in the monsoonal and Mediterranean-type climates of the world with very strong rainfall seasonality, typified by the Middle East, the African Sahel, the loess lands of China and the Mexican and Andean highlands. Only West Africa and the Amazon Basin have hints of early agriculture in relatively non-seasonal tropical climates like those of most of New Guinea, but for neither of these areas is there good evidence that the transition to agriculture was completely independent. However, the New Guinea highlands do have such evidence, thus making them interesting, even unique, for several reasons:

- The New Guinea transition was perhaps as old a transition as anywhere else in the world, and it was clearly pristine.

- It was focused on tree products, fruits and tubers, with no cereals (although sugarcane, like the cereals, is a member of the Gramineae family). The absence of cereals such as rice and foxtail millet (both native to China and normally cooked by boiling) is partly reflected in the absence of pottery in the New Guinea highland archaeological record, at least until recent centuries.

- In its early days, the New Guinea agricultural scene was without any domestic animals, until the pig was introduced from Indonesia, directly or indirectly, shortly before 3000 years ago, with dog and chicken perhaps later.

- The New Guinea region of early agriculture was relatively low in overall food productivity because of the absence of cereals and domestic animals. It did not produce the huge urban populations or continental-scale expansions of languages and societies that occurred out of some of the other regions, such as the Middle East, China, Mesoamerica and the northern Andes. On the other hand, population expansions within New Guinea and from the Near Oceanic region into those regions of Island Melanesia that lie beyond the Solomon Islands have undoubtedly occurred during the past 3000 years, during which time both agriculture and advanced navigational skills existed in coastal areas.

7 See Bellwood 1997 and Bellwood and Dizon 2008. Lipson et al. (2014) give an excellent presentation of current autosomal genetic evidence for the spread of Austronesian-speaking populations in Island Southeast Asia. 
- Early agriculture in the New Guinea highlands involved the digging of channels to control water level, thus representing an interesting and unusual investment in technology. However, there is disagreement amongst the investigators about the time of its appearance, with estimates for the earliest artificial ditching ranging from 10,000 years ago by Hughes and Golson to only 4000 years ago by Denham, who argues that earlier channels were natural, not artificial (Denham, Golson and Hughes 2004: 293; see Chapters 11-13 here). Over subsequent millennia, characteristic New Guinea systems of agricultural intensification were developed in order to feed growing populations and to circumvent problems with loss of soil nutrients (see Chapters 14-16 here). Overall, this process of intensification has been successful, assisted no doubt by the introduction of the high-yielding sweet potato in recent centuries. But it was also interrupted on a local scale from time to time by periods of fallow and land abandonment, such as those suggested by the periodicity within the Kuk sequence.

Perhaps we can understand some of the possible reasons for early agriculture in New Guinea by taking a comparative look at other parts of the world. In my own view, the transition to a cultivator lifestyle in the New Guinea highlands occurred, as in the Middle East, after the very marked and rapid climatic fluctuations that occurred over much of the world at the end of the Pleistocene Ice Ages. In the Middle East, the situation of increasing plenty combined with periodic oscillations promoted moves towards food production. I suspect a similar trajectory in New Guinea, where the agricultural developments occurred especially in those highland regions in which many plants, such as taro, banana and sugarcane, would have moved upwards in altitude towards the limits of their growth ranges with the climatic amelioration. Such marginal locations would have favoured fluctuating food supplies as climates continued to oscillate back and forth, even with decreasing amplitude, from warmer to colder (with periodic frost), and perhaps also from wetter to drier. Such changes could have promoted the planting of useful species in protected areas (e.g. away from frost, or in swamps) in order to improve supply. This view focuses on the 'edge of the range' as a location where fluctuation can promote innovation. ${ }^{8}$

Some archaeologists do not agree with the above reconstruction, which implies, firstly, that fairly rapid changes in lifestyle occurred during the early Holocene and, secondly, that New Guinea agricultural origins occurred in the highlands rather than the lowlands. On the first issue, others (Groube 1989; Denham and Barton 2006) hold the view that New Guinea agriculture was the culmination of a much more drawn-out and continuous process of plant management, beginning with forest clearance soon after initial human settlement more than 40,000 years ago. In this regard, according to David Rindos (1984), plants have co-evolved with humans for as long as they have been exploited by them, adapting to the novel dispersal mechanisms provided as a result of human patterns of collection and discard. However, despite his gradualist views, Rindos (1984: 143,191) still recognised that actual transitions to conscious cultivation (which he termed 'agricultural domestication') occurred in the various regions of early agriculture, perhaps rapidly, and probably driven by population increase. Co-evolution need not necessarily mean constant and gradual change through time, with no revolutionary episodes. In my view, the environmental changes in the New Guinea highlands after the end of the last glaciation were as rapid as and of equal magnitude to those in other parts of the world and I see no reason why New Guinea should necessarily have taken a different or more gradual trajectory towards agriculture than other regions.

On the second issue, whether agriculture in New Guinea began in the lowlands or in the highlands, my current opinion favours a highlands origin and in this regard I am in agreement with Denham, Haberle and Lentfer (2004). The highlands provide both archaeological evidence and a persuasive early Holocene environmental background for indigenous agricultural origins.

8 As proposed for the New Guinea highlands by Bellwood 1996: 485-486 and Haberle and Chepstow-Lusty 2000. 
The New Guinea lowlands at present provide neither, although a spread of agriculture from the highlands into the lowlands, for instance down the Markham Valley or into the Sepik Basin, before the arrival of Austronesian-speaking populations in coastal New Guinea about 3000 years ago is very likely, in my view. Such an early spread of agricultural practices involving tuber cropping and arboriculture into coastal lowlands and adjacent islands would go far towards explaining why Austronesian settlement in New Guinea has been such a tentative phenomenon. The indigenous Papuan-speaking food producers spread first, at least in theory.

\section{Some final observations}

The degree of continuity of the human population from initial settlement to the present appears to have been very high in the region of western Melanesia generally, and especially in the New Guinea highlands. There are no signs of major population or language replacements from external sources during the Holocene, although internal expansions as a result of the acquisition of systematic agriculture are very likely, particularly from the perspective of linguistic evidence pertaining to the Trans New Guinea Family of languages. ${ }^{9}$ There is no evidence of population replacements from outside penetrating the interior of New Guinea, which suggests that agricultural innovation has been a successful process there and that it has been driven from within, not from without. The ancestors who dug the sequence of Kuk ditches must take at least some of the credit for this.

So, what is special about New Guinea? Certainly, special features of the natural and cultural environment of the New Guinea highlands include closeness to the equator, providing a unique equatorial non-seasonal and cool tropical climate in world terms. There is also the factor of technological continuity, in archaeological terms, from the Palaeolithic to recent ethnographythere is no pottery, and ground axes are the only concessions to 'Neolithicisation'. As I have stated (Bellwood 2005b: 10) in reviewing the book Papuan Pasts (Pawley et al. 2005):

To understand why New Guinea is so special we have to begin by thinking geographically. Open an atlas. At first sight, New Guinea looks just like an easterly extension to Indonesia, and one of the sad results of recent colonial hubris is that the western half of it indeed still belongs to Indonesia, while the other (the eastern part) forms the independent nation of Papua New Guinea. Yet, a closer look at the atlas will reveal that New Guinea is not a part of Indonesia in any geological sense, let alone any fundamental ethnological sense. As the northern landmass of the Greater Australian continent it is actually colliding with the eastern end of the Sunda-Banda volcanic arc, forcing back the latter into a sinuous retreat and in the process heaving its own continental rocks into a massive island-long cordillera of folded mountains that in places reach over 4500 metres in altitude. Any traveller can see at a glance that the chained but separate volcanoes of Indonesia bear little resemblance to the alpine structure of New Guinea. This is an island that is absolutely unique in geomorphological terms, with an equatorial location and a relatively iron-bound cordillera, with fertile hidden valleys between about 1400 and 1850 metres above sea level that the rest of the world did not discover until the 1930s. This cordillera is the clue to New Guinea's significance in prehistory and its achievement of indigenous agriculture early in the Holocene.

The excavations at Kuk give us a remarkable record of at least 6000 years of agriculture and swamp management in the New Guinea highlands, one so far unique in the world. To fill out this picture, and improve our understanding further, we need additional archaeological records similar to that from Kuk, especially for the first half of the Holocene. So far this exists mainly in limestone caves, places hardly well located to throw light on agricultural developments and the growth of sedentary villages. It is to be hoped that future social conditions in both halves of $\mathrm{New}$ Guinea will allow research to proceed in safety and a context of indigenous community goodwill.

9 On this issue see Pawley 2005: 97-99; Ross 2005: 41; Pawley 2007; cf. Denham 2005a: 351 and Golson 2005: 474. 
This text is taken from Ten Thousand Years of Cultivation at Kuk Swamp in the Highlands of Papua New Guinea, edited by Jack Golson, Tim Denham, Philip Hughes, Pamela Swadling and John Muke, published 2017 by ANU Press, The Australian National University, Canberra, Australia. 